\title{
Attractant Role of Bacterial Bioluminescence of Photorhabdus/uminescenson a Galleria mellonella Model
}

\author{
Walter Patterson ${ }^{1}$, Devang Upadhyay ${ }^{1,}{ }^{*}$, Sivanadane Mandjiny ${ }^{2}$, Rebecca Bullard-Dillard ${ }^{3}$, \\ Meredith Storms ${ }^{4}$, Michael Menefee ${ }^{5}$, Leonard D. Holmes ${ }^{1}$ \\ ${ }^{1}$ Sartorius Stedim Biotechnology Laboratory, Biotechnology Research and Training Center, The University of North Carolina at Pembroke, \\ Pembroke, USA \\ ${ }^{2}$ Department of Chemistry and Physics, The University of North Carolina at Pembroke, Pembroke, USA \\ ${ }^{3}$ School of Graduate Studies and Research, The University of North Carolina at Pembroke, Pembroke, USA \\ ${ }^{4}$ College of Arts \& Sciences, The University of North Carolina at Pembroke, Pembroke, USA \\ ${ }^{5}$ Thomas Family Center for Entrepreneurship, The University of North Carolina at Pembroke, Pembroke, USA
}

Email address:

danny.uncp@gmail.com (D. Upadhyay)

\section{To cite this article:}

Walter Patterson, Devang Upadhyay, Sivanadane Mandjiny, Rebecca Bullard-Dillard, Meredith Storms, Michael Menefee, Leonard D. Holmes. Attractant Role of Bacterial Bioluminescence of Photorhabdusluminescenson a Galleria mellonella Model. American Journal of Life Sciences. Vol. 3, No. 4, 2015, pp. 290-294. doi: 10.11648/j.ajls.20150304.16

\begin{abstract}
Though the intricate relationship between the entomopathogenic nematode Heterorhabditis bacteriophora and its symbiotic bacterial counterpart Photorhabdus luminescensis generally known; the role of bioluminescence produced by the bacterial symbiont is yet to be identified. The objective of this study was to determine if bacterial luminosity plays a crucial role in attraction of larval insect hosts. This study focused on bacterial bioluminescence produced from both in vitro and in vivo culturing of the bacterial symbiont. The obtained results portrays that the average distance between Galleria mellonellalarvae and the bacterial light source ( $P$. luminescens)decreased in a linear fashion as a function of increasing intensities of luminosity; thereby supporting the hypothesis that bioluminescence offers a symbiotic role to attract insect host larvae.
\end{abstract}

Keywords: Photorhabdus Luminescens, Heterorhabditis Bacteriophora, Symbiosis, Bioluminescence, Biological Control Agent

\section{Introduction}

Heterorhabditis bacteriophora is an entomopathogenic nematode (EPN)that is currently being explored as a biological control agent for particular crop insect pests. $H$. bacteriophora is a cruiser species of nematode, meaning that infective juveniles "seek" out their insect host. In other words, populations of new infected juveniles that are generated from an infected host will exit the infected carcass and disseminate into the surrounding environment in search of anotherinsect host. This predation strategy is rather different than the ambush method which is employed by the sister genus Steinernema[1,2]. During the ambush method, infective juveniles of Steinernema remain sessile in a single location until an appropriate host is detected.

EPNs arelethal to the insect host due to the transmission of symbiotic entomopathogenic bacteria [3]. H. bacteriophora is associated with its bacterial symbiont
Photorhabdusluminescens [4]. The bacterial symbiont is carried within the gut of $H$. bacteriophorauntil the nematode reaches the hemolymph of an insect larva. Upon signaling, $H$. bacteriophoraexpels the bacterial symbiont, resulting in bacterial proliferation that quickly consumes available nutrients within the insect. During this time, the bacterial symbiontbegins to secrete digestive enzymes and insecticidal toxinsthat result in insect death within 24-48 hours [5, 6]. Additionally,P. luminescens and other sister species produce bioluminescence; which to date, are the only knownterrestrialbacterialcapable of doing so [1].A possible symbiotic role for bioluminescence is that nematodes exploit this mechanismas a way to attract host insects to the infected carcass. As a result, this mechanism would greatly increase survival of the nematode-bacterium complex.

Galleriamellonella is typically used as a model host 
organism to study nematode efficiency and bacterial toxicity. $G$. mellonella is also employed for in vivo culturing of EPNs and bacterial symbiontsfor laboratory use[7]. In relevance to this study, larvae of G.mellonella use 4 small organs (i.e. stemmata) on each side of its head in order to perceive lowquality vision. Stemmata contain light sensitive pigments (i.e. rhodopsins) that enable insect larvae to detectlight when excited [8]. G. mellonella rhodopsins havea lambda max (510 $\mathrm{nm})$, proximal to the peak wavelength $(490 \mathrm{~nm})$ ofthe bioluminescence produced by of $P$. luminescens $[8,9]$. Many other Lepidopteran species have rhodopsins whose lambda max falls within the same spectrum of bioluminescenceof $P$. luminescenssuch as Manducasexta and Spodopteraexigua(520nm and $515 \mathrm{~nm}$, respectively) $[9,10]$. The relatively small distances between emission peaks and insect rhodopsins may not have a significant impact on light detection within insect larvae.

In this study, larvae of G. mellonella were used to observe the effects of luminosity produced by $\mathrm{P}$. luminescens on insect attraction. This study was performed utilizing two different bacterial culturing conditions to producein vitro and in vivo light sources. These light sources, along with live, uninfected insect larvae, were placed into a gridded chamber and left in low external light conditions. Distances between light sources and live insects were determined from digital images and plotted against the corresponding luminosity reading. The results of this study support the idea that bacterial luminosity does attract insect larvae. Furthermore, this research suggests that bacterial luminosity is a symbiotic trait that infective juveniles of $\mathrm{H}$. bacteriophora exploit to attract insect hosts.

\section{Materials and Methods}

\subsection{Preparation of Materials and Supplies}

Infective juveniles of $H$. bacteriophora were obtained through in-house mass production protocols. P. luminescens cultures were isolated from infected $G$. mellonella using the method of Inman III and Holmes [11]. G. mellonella were obtained from Carolina Biological Supply Company (Burlington, North Carolina, USA). Using an inoculation loop, samples of infected hemolymph were aseptically obtained and streaked for bacterial isolation onto nutrient $\operatorname{agar}(\mathrm{g} / \mathrm{L}$ : beef extract, 6; digested gelatin, 10; and agar, 15).

$P$. luminescens was cultured overnight at $28^{\circ} \mathrm{C}$ and isolated colonies werethen subcultured onto a second plate. Isolated colonies from the second plateweretransferred to tubes containing $3 \mathrm{~mL}$ ofnutrient broth $(\mathrm{g} / \mathrm{L}$ : beef extract 6 ; digested gelatin, 10) and incubated at $150 \mathrm{rpm}$ and $28^{\circ} \mathrm{C}$ overnight. Following incubation, the liquid culture was scaled up to $50 \mathrm{ml}$ nutrient broth in a $250 \mathrm{~mL}$ Erlenmeyer flask. Cultures were incubated at $28^{\circ} \mathrm{Cand}$ agitated on an orbital shaker at $150 \mathrm{rpm}$ overnight. Unused infected Gallerialarvae from this step were used for in vivo luminosity studies.

Behavior chambers used in this experimentwere made from $100 \mathrm{~mm}$ x 20mm cell culture dishes (Corning, Inc.) and to them PetriStickers ${ }^{\mathrm{TM}}$ containing32 square gridswere affixed to the top for determining insect positions.Inside chamberswere lined with pre-moistened $9 \mathrm{~cm}$ Whatman filter paper and assembled. Chamber tops were drilled with four equidistant holes $(\sim 2 \mathrm{~mm}$ in diameters $)$ to allow gas exchange.

\subsection{Methods}

\subsubsection{Bacterial and Larvae Sampling}

G. mellonellawere screened for signs of infection based on melanization (darkening of pigment) and motor defects based on lack of response to physical stimulation. Larvae who did not display any of the traits where separated from the population received.

P. luminescens isolates where screened for contamination and phase variation. Contamination was screened for by gram-staining at transferring to new media. Phase variation was screened by only selecting cultures with relatively high bioluminescence.

\subsubsection{Exposure to Invitro Bioluminescence}

Forin vitro trials, $1 \mathrm{~mL}$ aliquots of the $50 \mathrm{~mL}$ overnight culture of $P$. luminescens culture were obtained and dispensed into $1.5 \mathrm{mLmicrocentrifuge} \mathrm{tubes.} \mathrm{Luminosity} \mathrm{of} \mathrm{sample}$ aliquots was measured with a Modulus ${ }^{\mathrm{TM}}$ single tube luminometer (Turner Biosystems) and reported in terms of relative luminosity units (RLU). Afterwards, each culture tube was placed at theperipheryof each chamber. HealthyG.mellonellalarvae were added carefully to the center of each chamber and placed in a lowlight environment for 10 minutes. After the 10 minute period, each chamber was briefly exposed to laboratory light and a digital photograph was taken at a fixed $90^{\circ}$ angle to the plate surface. Controlsconsisting of sterile culture broth,were also performed in the same manner.

\subsubsection{Exposure to in Vivo Bioluminescence}

$H$. bacteriophora - P. luminescens infected larvae of G.mellonella were measured for luminosity. The infected and highly luminescent carcasses of $G$. mellonella were placed at the peripheral of each chamber. Healthy, non-infected $G$. mellonellawere added to the center of each chamber. Methodology of in vivo testing was conducted in the same manner as in vitro testing, with dead, suffocated larvae acting as a control, which were discarded upon melanization.

\subsubsection{Analyses of Images}

Utilizing the known distances ofthe grid and the number of pixelsalong the grid from the digital photograph were used to determine ratios of pixels todistance. From the pixel grid,the location of the culture and the head of the G. mellonellawere found, thenumber of pixels separating those locations was then counted.The pixels to millimeter ratio of the individual photographs were used to calculate the distance of the Galleria larvae to the light source based on the number of pixels separating them. 


\section{Results}

\subsection{Effects of Bioluminescence from in Vitro Cultures on Galleria Mellonella}

Gallerialarvae exposed to in vitro cultures of $P$. luminescenstend to follow a set pattern of behavior towards luminosity. Results indicate that distancesbetween luminescent cultures and larval heads decrease as luminosity increases. When the RLU of the culture increase, larvaetend to move closer to the tube in aninversely linear fashion. This observation suggests that at higher intensities of bioluminescence attraction of the insect is greater; thereby reducing the distance between the insect and the luminescent culture. The slope produced from the in vitro data indicates an attraction rate of $-7.54 \mathrm{~mm} / 10^{5} \mathrm{RLU}$ as shown in Figure 1.

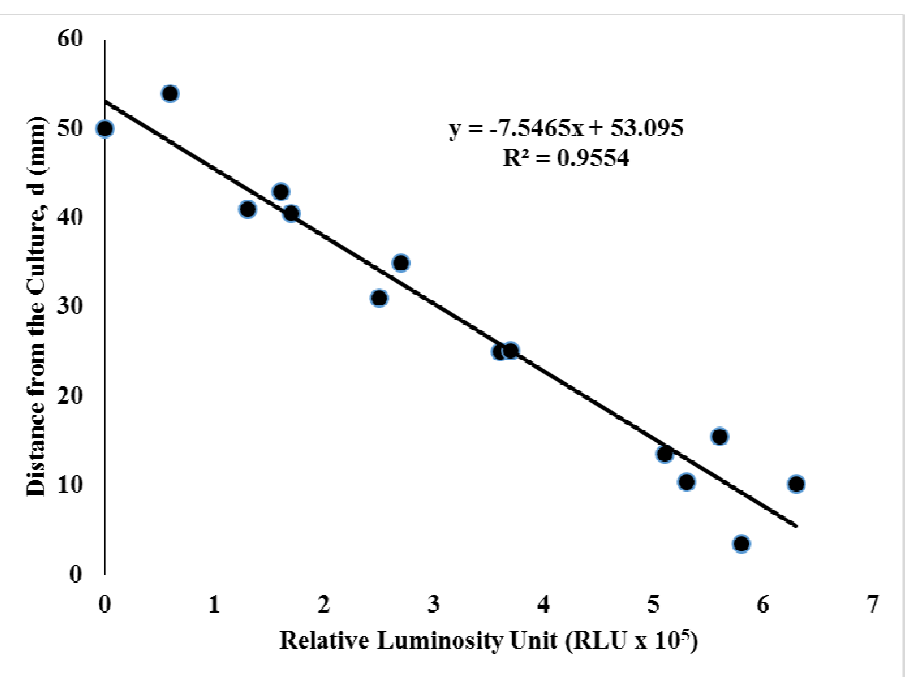

Figure 1. Effects of in vitrobioluminosity on Galleria mellonella position relative to P. luminescens culture. The overall trend ofin vitro culturing depicts an inversely linear relationship. The linear portion depicts an attractionrate of $-7.54 \mathrm{~mm} / 10^{5} \mathrm{RLU}$.

\subsection{Effects of Bioluminescenceproduced from in Vivo Cultures on Galleria Mellonella}

G.mellonella exposed to in vivo culturing of $P$. luminescens and $H$. bacteriophora within carcasses of otherlarvae were foundto display a similar inversely linear correlation between RLU and distances to carcasses. With the in vivo testing there is a linear trend that exhibits a decreasing distance to the carcass as RLU of in vivocultures increase (Figure 2). The rate of this linear trend was at -0.75 $\mathrm{mm} / 10^{5}$ RLU.During the period ofin vivoexperimentation $(\sim 5$ days), no healthy Galleriasubjects were infected by $H$. bacteriophora. Thissuggests that more incubation time is needed to force the emergence of infective juvenile nematodes from insect carcasses.

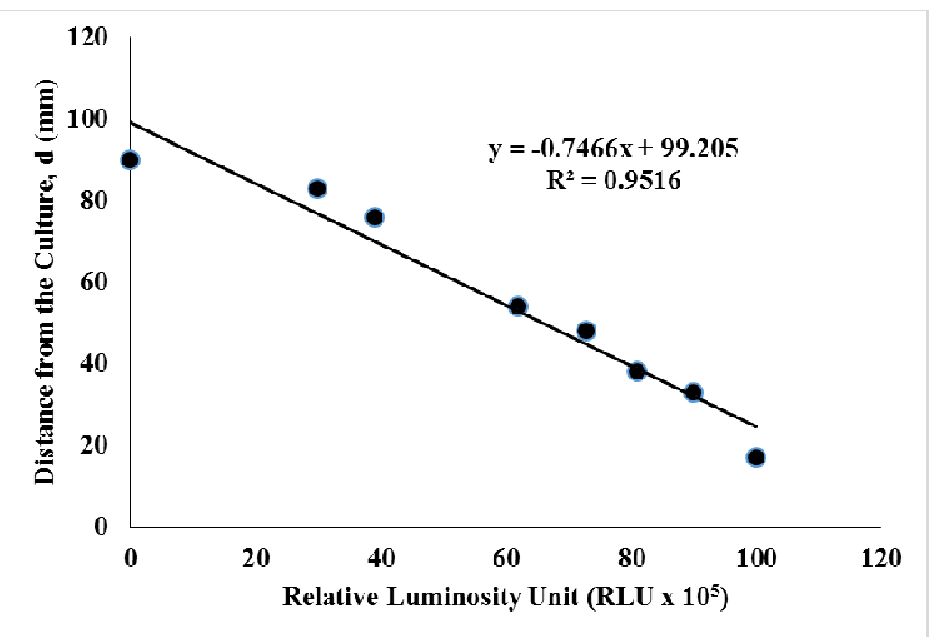

Figure 2. Experimental correlation between distance and RLU determined during in vivo trials. A linear correlation is observed that depicts a decreasing rate of $-0.75 \mathrm{~mm} / 10^{5} \mathrm{RLU}$ which is highly similar to the rate obtained through in vitro trials.

\section{Discussion}

H. bacteriophora is classified as a cruiser species and appears to conflict with the role of bioluminescence from $P$. luminescens as a tool for host attraction[2,12].The differentiation between cruiser and ambush EPNs is based on 
the observation of species' microscopic movements, rather than their macroscopic distribution in an ecosystem.This conflict can be resolved by considering the relative effectiveness of $P$. luminescens on G.mellonella, and its ability to attract larvae from portions of the environment in which $H$. bacteriophoradoes not inhabit.From this study, onlyhigh luminosityreadings, both in vitro and in vivoculturing systems,show effective attraction of G.mellonella larvae. The possible explanation of the role of bacterial bioluminescence is that at high RLU, host insects are attracted to a certain distance from the infected insect carcass. Once infective juveniles (IJs) of $H$. bacteriophoraemergefrom the carcass, they wouldmobilize within the environment to detect and attack baited hosts. Ultimately, luminosity draws insect hosts closer to the host cadaver to reduce the distance nematode would have to travel before attacking a new host and as a consequence, survival of both $P$. luminescens and $H$. bacteriophora is greatly increased.

Both culturing methods resulted in linear curvesthat depicted decreasing distancesbetween insect and bacterial cultures as luminosity increased. These trends indicate that highin vivo luminosity would be expected in a natural setting that issufficient to attract larval insect hosts. The differences in slopes could be due to secondary metabolites or other compounds produced by $P$. luminescens. Researchwith ant models has shown, that $P$. luminescenssecretes a secondary metabolite that repeals insects from the host cadaver. This offers protection to their nematode counterparts from being consumed by other organisms [13]. Controls from both in vivo and in vitro tests may provide some evidence in support of this. When $G$. mellonellalarvae were exposed to nonluminescent in vitro cultures of $P$. luminescens (Phase II), larvaewere found to have an average distance $(\sim 50 \mathrm{~mm})$ from the non-luminescent culture. On the other hand, in vivo control larvae exposed todead non-luminescent larvae were found at the furthest distance away from the cadaver $(\sim 100 \mathrm{~mm})$.

\section{Conclusion and Recommendations}

The results of this experiment demonstrate that bioluminescence produced by $P$. luminescens can attract healthy insect larvae towards an infected host. This is evidenced by the employment of $G$. mellonella larvae and measuring the distancebetween luminescent cultures and larval insect heads as a relation to increasing luminosity. The results of bioluminescence enable a greater probability that healthy larvae will become infected by attraction to the infectious host carcass. This experiment concludes that bioluminescence produced by $P$. luminescensmay be used to attract new insect hosts and as a consequence, $H$. bacteriophora nematodes benefit by the reduction of distance to infect another insect host.Further studies are required to determine the nature of the relationship between the attraction and repulsion of $G$. mellonellato bioluminescence and secreted bacterial compounds, respectively, produced by P. luminescens.
There are two mechanisms by which Galleria can be infected. Cruisingnematodesmay utilizebioluminescence to expand its range.This study certainly demonstrates that $P$. luminescens will attract and infect $G$. mellonella. However, the study does not eliminate the possibility that Galleriamay also become infected through contact with other infected larvae (dead or alive).Further study may provide evidence that bioluminescent surface-sanitized G. mellonellacanalso infect insect larvae.

\section{Acknowledgments}

Financial support was provided, in part, by the: Farm Bureau of Robeson County, North Carolina; University of North Carolina at Pembroke (UNCP) Office of the Provost and Academic Affairs; UNCP Department of Chemistry and Physics; North Carolina Biotechnology Center (NCBC) and UNCP Thomas Family Center. Special thanks are given to Dr. William "Bill" Brandon and Mr. Edward Derosier for technical consultations during preliminary studies involving simulated light.

\section{References}

[1] Georgis R, Gaugler R (1991) Predictability in biological control using entomopathogenic nematodes.J Econ Entomol $84: 713-720$

[2] Ehlers RU (1992)Environmental and biotic factors influencing the control potential of entomopathogenic nematodes of the genus Steinernema and Heterorhabditis. In:Gommers F J, Mass PWT (eds) Nematology from Molecule to Ecosystem. European Society of Nematologists,UK, pp201-212

[3] Ciche TA, Darby C, Ehlers RU,ForstS, Goodrich-Blair H (2006)Dangerous liaisons: The symbiosis of entomopathogenic nematodes and bacteria. Biol Control $38: 22-46$

[4] Fischer-Le SauxM, Viallard V, Brunel B, Normand P, Boemare NE(1999)Polyphasic classification of the genus Photorhabdus and proposal of new taxa: P. luminescens subsp.luminescens subsp. nov.,P. luminescens subsp. akhurstii subsp. nov., P. luminescens subsp.laumondii subsp. nov., P. temperata sp. nov., P. temperata subsp. temperata subsp. nov.and P. asymbiotica sp. nov. Int J SystEvolMicr49:16451656

[5] Hinchliffe S, Hares M, Dowling A, ffrench-Constant RH (2010)Insecticidal Toxins from the Photorhabdus and Xenorhabdusbacteria. Open Toxicol J 3:101-118

[6] DabornPJ, Waterfield N, Silva CP, Au CPY, Sharma S, ffrench-ConstantR H(2002)A single Photorhabdus gene, makes caterpillarsfloppy ( $m c f)$, allows Escherichia coli topersist within and kill insects. P NatlAcadSci USA 99:10742-10747

[7] $\mathrm{Hu} \mathrm{K}$ and Webster J (2000) Antibiotic production in relation to bacterial growth and nematode development in Photorhabdus Heterorhabditis infected Galleria mellonella larvae. FEMS Microbiology Letters 189:219-223 
[8] Pande C, Deng H, Rath P, Callender RH, Schwemer J(1989) Resonance raman spectroscopy of an ultraviolet-sensitive insect rhodopsin.Biochemistry-US 26:7426-7430

[9] Welham P, Stekel D(2009)Mathematical model of the lux luminescence system in the terrestrial bacterium Photorhabdusluminescens. Mol Biosyst5:68-76

[10] Briscoe A, Bernard G (2009) Eye shine and spectral tuning of long wavelength-sensitive rhodopsins: no evidence for redsensitive photoreceptors among five Nymphalini butterfly species. J ExpBiol208:687-696
[11] Inman III FL, Holmes LD (2012)The effects of trehalose on the bioluminescence and pigmentation of the phase I variant of Photorhabdus luminescens. J Life Sci6:119-129

[12] Inman III FL, Singh S, Holmes LD (2012) Mass production of the beneficial nematode Heterorhabditis bacteriophora and its bacterial symbiont Photorhabdus luminescens. Indian $\mathrm{J}$ Microbiol 52:316-324

[13] Zhou X, Kaya HK, Heungens K, Goodrich-Blair H (2002) Response of ants to a deterrent factor(s) produced by the symbiotic bacteria of entomopathogenic nematodes. Appl Environ Microbiol 68:6202-6209 